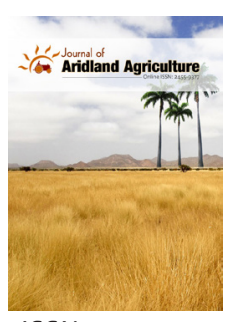

ISSN: $2455-9377$

Received: April 01, 2021 Revised: May 18, 2021 Accepted: May 19, 2021 Published: June 04, 2021

*Corresponding authors: Sang Un Park; E-mail:supark@cnu.ac.kr Sook Young Lee;

E-mail: seedbank@chosun. ac.kr

\section{Analysis of volatile compounds from three species of Atractylodes by Gas Chromatography-Mass Spectrometry}

\author{
Jin Piao', Soon Sung Lim², Haeng Hoon Kim³, Sook Young Lee,*, \\ Sang Un Park ${ }^{5, *}$
}

'Department of Agriculture, College of Agriculture, Yanbian University, Yanji, China, ${ }^{2}$ Department of Food Science and Nutrition and Institute of Natural Medicine, Hallym University, Chuncheon, 200-702, Korea, ${ }^{3}$ Department of Well-being Resources, Sunchon National University, Suncheon, Jeollanam-do 57922, Korea, ${ }^{4}$ Marine Bio Research Center, Chosun University, 61-220 Myeongsasimni, Sinji-myeon, Wando-gun 59146, Jeollanamdo, Korea, ${ }^{5}$ Department of Crop Science and Department of Smart Agriculture Systems, Chungnam National University, 99 Daehak-ro, Yuseong-gu, Daejeon 34134, Korea

\begin{abstract}
A total of 99 different volatile compounds were detected through Gas Chromatography-Mass Spectrometry (GC-MS) from three species of Atractylodes, namely Atractylodes lancea, Atractylodes japonica, and Atractylodes chinensis. Thirteenvolatile flavor compounds i.e. acid, alcohol, aldehyde, alkane, alkene, alkyne, ester, ketone, monoterpene, oxygenated monoterpene, sesquiterpene, oxygenated sesquiterpene, and oxygenated triterpenoid detected from different species of Atractylodes. It was observed that all the species contained 38 common compounds, while A. lancea contained 7 unique compounds, A. japonica has 4 unique compounds, and A. chinensis hold 6 compounds not detected in the other extracts. In addition, essential oils from A. lancea and A. japonica possessed 11 compounds in common, and A. lancea and A. chinensis possessed 19 compounds in common. The remaining 14 compounds were detected only in A. japonica and A. chinensis. The total content of all components in the species was comparable, with $82.528 \%$, $81.766 \%$, and $81.799 \%$ of volatile components being detected for A. lancea, A. japonica, and A. chinensis, respectively Curzerene was found to be the most predominant compound in both A. lancea (14.1\%) and A. chinensis (16.7\%), while murolan-3,9(11)-diene-10-peroxy was found predominantly in A. japonica (16.8\%). The present study suggests that the identified volatile compounds may possess important biological properties, and could be suitable for application in both oriental medicines and the pharmaceutical industry.
\end{abstract}

KEYWORDS: Volatile compounds, medicinal plants, Atractylodes chinensis, Atractylodes japonica, Atractylodes lancea, essential oil, GC-MS analysis

\section{INTRODUCTION}

Volatile organic compounds (VOC), generally lipophilic liquids with high steam pressures, symbolize the largest group of natural products in plants. These types of compounds cover multiple effects on both floral and vegetative tissues in many plant species (Pichersky et al., 2006). Usually, much floral volatiles provides to attract pollinators and also do something as guards for precious reproductive parts of plants against harmful pathogens, parasites, and herbivores (Dudareva et al., 2004). In most cases, vegetative volatiles engages in the signaling of interplant or inner plant organs and plant defense against pathogens, heat, and oxidative stress (Unsicker et al., 2009). In addition, numerous aromatic plants have been used as flavorings, preservatives, and herbal remedies (Pichersky et al., 2006).
Atractylodes is one of an important genus belongs to the family Asteraceae and is composed of eight species of perennial medicinal plants widely distributed in East Asia (Willis, 1966). Some species of the Asteraceae family, including Atractylodes lancea, A. japonica, and A. chinensis, are well known for their use in traditional Chinese medicine. Essential oils are the main active constituents of Atractylodes spp., with previous studies examining volatile oil biosynthesis in some species of Atractylodes. It was reported that one of the endophytic Acinetobacter sp. ALEB16A enhanced the biosynthesis of volatile components in A. lancea (Wang et al., 2015). Inoculation with the endophytic fungus of Gilmaniella sp. AL12 boosted the actions of total protein phosphorylation needed for endophyteinduced volatile oil production in A. lancea (Ren and Dai, 2012). It proclaimed that jasmonic acid performs in NO- and $\mathrm{H}_{2} \mathrm{O}_{2-}$

Copyright: $\odot$ The authors. This article is open access and licensed under the terms of the Creative Commons Attribution License (http://creativecommons.org/licenses/by/4.0/) which permits unrestricted, use, distribution and reproduction in any medium, or format for any purpose, even commercially provided the work is properly cited. Attribution - You must give appropriate credit, provide a link to the license, and indicate if changes were made. 
mediated volatile oil accumulation as a downstream signaling molecule in A. lancea provoked by endophytic fungi (Ren and Dai, 2012). The existence of a fungal elicitor might therefore considerably enhance the content of volatile oil in A. lancea (Zhang et al., 2008). Furthermore, it was reported that the geographical disparity for the active components in rhizome essential oils of A. lancea and A. chinensis imitates mainly for genetic variability (Takeda et al., 1996).

It was also reported that essential oil from Atractylodes spp. showed an insecticidal activity and postponed gastric emptying in stress-induced rats (Zhang et al., 2008). The governing actions on delayed gastric emptying from the essential oil in Atractylodes lancea are mostly owing to the reduction of the release of the central corticotropin-releasing factor (Zhang et al., 2008). Besides, these essential oil from Atractylodes chinensis (DC.) Koidz showed strong insecticidal activity which works well against Drosophila melanogaster L. (Chu et al., 2011). It has been previously reported that plants belonging to the Atractylodis genus are rich in volatile compounds and essential oils, including sesquiterpenes and polyacetylenes. In particular, A. lancea is used in Chinese patent medicines (Chen et al., 2007; Xie et al., 2008). These essential oils and volatile compounds can be used in antiinflammatory, anticonvulsant, sedative, analgesic, antianoxic, antiviral, and anti-hepatotoxic treatments (Guo et al., 2006).

As these species showed lots of inevitable biological properties, therefore this study was undertaken to identify different volatile components present in A. lancea, A. japonica, and A. chinensis, and compared the characteristics of each component using gas chromatography-mass spectrometry.

\section{MATERIALS AND METHODS}

\section{Identification of Different Volatile Compounds Through Headspace Solid-phase Microextraction Technique}

Three species of Atractylodeswere collected from a different area of China i.e. Atractylodes lancea Thunb. was collected from Wuhan, Hubei, Atractylodes japonica Koidz was from Yanji, Jilin, and Atractylodes chinensis Koidz was from Zhangjiajie, Hunan. After collection, samples were weighed and immediately taken into a vial containing a headspace of $25 \mathrm{~mL}$. For the absorption of volatile compounds, a fused-silica fiber coated having a $75 \mu \mathrm{m}$ layer of carboxen/poly dimethyl siloxane (CAR/PDMS) was utilized. The fiber was opened to the headspace of the vial maintaining $25^{\circ} \mathrm{C}$ for about $20 \mathrm{~min}$, after that it was eliminated from the vial and initiated directly into the GC injector. Here thermal desorption analysis was conducted at $250{ }^{\circ} \mathrm{C}$ for $3 \mathrm{~min}$. The compounds were identified from their mass spectra and the quantitative detection was calculated by utilizing peak areas of the compounds. Standards for GC-MS were collected from the National Institute of Standards and Technology (NIST).

\section{GC and GC-MS Analysis}

Herewith an Agilent 6890N GC mainframe connected with an HP-5 fused-silica capillary column $(30 \mathrm{~m} \times 0.32 \mathrm{mmID}, 0.25 \mu \mathrm{m}$ film thickness), as well as the flame ionization detector (FID) (Agilent, USA) the GC analysis was done. Temperatures of both injector and detector for each analysis were placed at $250^{\circ} \mathrm{C}$ and $280{ }^{\circ} \mathrm{C}$, respectively. As the carrier gas, here nitrogen was used maintaining a flow rate of $1.0 \mathrm{ml} / \mathrm{min}$. The column temperature was retained at $50^{\circ} \mathrm{C}$ for $5 \mathrm{~min}$ and was then modified as follows: 1) Ramp from $50{ }^{\circ} \mathrm{C}$ to $260^{\circ} \mathrm{C}$ at a rate of $3{ }^{\circ} \mathrm{C} \mathrm{min}{ }^{-1}$; 2) Ramp from $260{ }^{\circ} \mathrm{C}$ to $280{ }^{\circ} \mathrm{C}$ at a rate of $10^{\circ} \mathrm{C} \mathrm{min}-1$; 3) Hold at $280{ }^{\circ} \mathrm{C}$ for $5 \mathrm{~min}$.

GC-MS analysis was conducted on GC/MSD Polaris Q (Thermo Finnigan, USA) prepared with an HP-5 fused-silica capillary column ( $30 \mathrm{~m} \times 0.32 \mathrm{~mm}$ ID, $0.25 \mu \mathrm{m}$ film thickness) (Agilent, USA). Here as the carrier gas, Helium was utilized giving a flow rate of $1.0 \mathrm{mLmin}^{-1}$. An electron ionization system having a $70 \mathrm{eV}$ system energy, a $250 \mu \mathrm{A}$ trap current, and an ion source at $200{ }^{\circ} \mathrm{C}$ was used for GC-MS detection.

\section{Samples Identification}

The respective compounds were identified by contrasting the mass spectra also with the data of NIST and WILLY library of the GC-MS system as well as the data from the literature. Total ion current chromatograms were measured following the mass range 40-400amu.

\section{RESULTS}

\section{Composition of Essential Oil}

GC-MS analysis assisted to detect 99 volatile compounds from three different species of Atractylodes by evaluating their GC-MS spectra with standard compounds as well as with previous literature reports (Yosioka et al., 1976; Chen et al., 2009; Wang et al., 2012). A total of 99 volatile compounds were detected in A. lancea, A. japonica, and A. chinensis (Table 1). Among the 99 volatile compounds, six compounds, i.e. 3-octen-5-yne, 5-butyl-1,3-cyclohexadiene, caryophyllene, cubenol, 1,2,3,3a,4,5,6,7-octahydro-1,4dimethyl-7-(1-methylethenyl)-[1R-(1 $\alpha, 3 \mathrm{a} \beta, 4 \alpha, 7 \beta)]$-azulene, and $(3 \mathrm{aS}, 6 \mathrm{~S}, 6 \mathrm{aS}, 9 \mathrm{aR}, 9 \mathrm{bR})$-azuleno [4,5-b]furan-2,9-dione, were detected only in A. lancea. In the case of A. japonica, four unique compounds were detected i.e. $\beta$-curcumene, decahydro-1,5,5,8a-tetramethyl-[1s-(1 $\alpha, 3 \mathrm{a} \beta, 4 \mathrm{a} \alpha, 7 \beta, 8 \mathrm{a} \beta)]-1,4-$ methanoazulen-7-ol, 3-hydroxy-7-isopropenyl-1,4a-dimethyl2,3,4,4a,5,6,7,8-octahydronaphthalen-2-yl acetate, and methyl 9,11-octadecadienoate. In addition, six volatile compounds, namely $\alpha$-phellandrene, l-methyl-3-(1-methylethyl)-benzene, 6-isopropylidene-1-methyl-bicyclo[3.1.0]hexane, $\beta$-copaene, ledene oxide-(II), and longiverbenone were found only in A. chinensis. Furthermore, 11 of the 99 compounds, namely 2,5-dimethyl-3-methylene-1,5-heptadiene, $\alpha$-ethyl- $\alpha$-2,5,7octatrienyl-benzenemethanol, decahydro-1,1,3a-trimethyl7-methylene-[1aS-(1a $\alpha, 3 \mathrm{a} \alpha, 7 \mathrm{a} \beta, 7 \mathrm{~b})]$ - $1 H$-cyclopropa [a] naphthalene, 1-ethenyl-1-methyl-2,4-bis(1-methylethenyl)[1S-(1 $\alpha, 2 \beta, 4 \beta)]$-cyclohexane, $\alpha$-acorenol, $\gamma$-eudesmol, $1,2,3,4,4 a, 5,6,8$ a-octahydro- $\alpha, \alpha, 4$ a , 8-tetramethyl-[2R$(2 \alpha, 4 \mathrm{~A} \alpha, 8 \mathrm{~A} \beta)]$-2-naphthalenemethanol, neocurdione, 
Table 1: Volatile compounds and their quantities in Atractylodes spp., determined by solid-phase microextraction (SPME).

\begin{tabular}{|c|c|c|c|c|c|c|c|}
\hline \multirow[t]{2}{*}{ No } & \multirow[t]{2}{*}{ Compounds } & \multirow{2}{*}{$\begin{array}{c}\text { Retention } \\
\text { time } \\
\text { (min) }\end{array}$} & \multicolumn{3}{|c|}{ Peak area (\%) } & \multicolumn{2}{|c|}{ Class of chemical Chemical formula } \\
\hline & & & $\begin{array}{c}\text { Atractylodes } \\
\text { lancea } \\
\text { Thunb }\end{array}$ & $\begin{array}{c}\text { Atractylodes } \\
\text { japonica } \\
\text { Koidz }\end{array}$ & $\begin{array}{c}\text { Atractylodes } \\
\text { chinensis } \\
\text { Koidz }\end{array}$ & & \\
\hline 1 & 3-0cten-5-yne & 11.392 & 9.007 & - & - & Alkyne & $\mathrm{C}_{8} \mathrm{H}_{12}$ \\
\hline 2 & $\gamma$-Terpinene & 13.613 & - & 0.053 & 10.334 & Monoterpene & $\mathrm{C}_{10}^{8} \mathrm{H}_{16}^{12}$ \\
\hline 3 & D-Limonene & 13.801 & 0.585 & - & 0.198 & Monoterpene & $\mathrm{C}_{10}^{10} \mathrm{H}_{16}^{16}$ \\
\hline 4 & $\alpha$-Phellandrene & 14.447 & - & - & 0.211 & Monoterpene & $\mathrm{C}_{10}^{10} \mathrm{H}_{16}^{16}$ \\
\hline 5 & 5-Butyl-1,3-cyclohexadiene & 14.515 & 0.509 & - & - & Alkene & $\mathrm{C}_{10}^{10} \mathrm{H}_{16}^{16}$ \\
\hline 6 & 2-Carene & 15.615 & 2.273 & 0.426 & 0.113 & Monoterpene & $\mathrm{C}_{10}^{10} \mathrm{H}_{16}^{16}$ \\
\hline 7 & 1-Methyl-3-(1-methylethyl)benzene & 15.761 & - & - & 0.086 & Monoterpene & $\mathrm{C}_{10}^{10} \mathrm{H}_{14}^{16}$ \\
\hline 8 & $\alpha$-Ylangene & 16 & 0.748 & - & 0.534 & Sesquiterpene & $\mathrm{C}_{15}^{10} \mathrm{H}_{24}^{14}$ \\
\hline 9 & 2,5-Dimethyl-3-methylene-1,5-heptadiene & 16.483 & 0.091 & 0.239 & - & Monoterpene & $\mathrm{C}_{10}^{15} \mathrm{H}_{16}^{24}$ \\
\hline 10 & 4-Terpinenyl acetate & 17.028 & 0.463 & - & 8.205 & $\begin{array}{l}\text { Oxygenated } \\
\text { monoterpene }\end{array}$ & $\mathrm{C}_{12}^{10} \mathrm{H}_{20}^{16} \mathrm{O}_{2}$ \\
\hline 11 & 6-Isopropylidene-1-methylbicyclo[3.1.0]hexane & 17.218 & - & - & 1.698 & Monoterpene & $\mathrm{C}_{10} \mathrm{H}_{16}$ \\
\hline 12 & 1,5,5,6-Tetramethyl-1,3-cyclohexadiene & 17.502 & 0.245 & - & 0.156 & Monoterpene & $\mathrm{C}_{10}^{10} \mathrm{H}_{16}^{16}$ \\
\hline 13 & $m$-Phenethylbenzonitrile & 18.24 & 0.643 & 0.028 & 0.711 & Other & $\mathrm{C}_{15}^{10} \mathrm{H}_{13} \mathrm{~N}$ \\
\hline 14 & 1,2-Ethanediol monobenzoate & 18.498 & 0.086 & - & 1.318 & Ester & $\mathrm{C}_{9} \mathrm{H}_{10} \mathrm{O}_{3}$ \\
\hline 15 & 2-Bromoethyl benzoate & 18.696 & 0.096 & 0.953 & 0.115 & Ester & $\mathrm{C}_{9} \mathrm{H}_{9} \mathrm{BrO}_{2}$ \\
\hline 16 & $\alpha$-Ethyl- $\alpha$ - $(2,5,7$-octatrienyl) benzenemethanol & 18.811 & 0.171 & 0.135 & - & Alcohol & $\mathrm{C}_{17} \mathrm{H}_{22} \mathrm{O}$ \\
\hline 17 & 2,6-Pyridinedicarboxaldehyde & 19.202 & 0.155 & - & 0.302 & Aldehyde & $\mathrm{C}_{6} \mathrm{H}_{5} \mathrm{NO}^{2}$ \\
\hline 18 & $\begin{array}{l}\text { 3,6-Diethyl-3,6-dimethyl-trans-tricyclo[3.1.0.0(2.4)] } \\
\text { hexane }\end{array}$ & 21.164 & 0.193 & - & 0.647 & Alkane & $\mathrm{C}_{12}^{0} \mathrm{H}_{20}$ \\
\hline 19 & Longifolene-(V4) & 26.417 & 0.232 & - & 0.359 & Sesquiterpene & $\mathrm{C}_{15} \mathrm{H}_{24}$ \\
\hline 20 & $\begin{array}{l}\text { IR, } 4 \mathrm{R}, 7 \mathrm{R}, 11 \mathrm{R}-1,3,4,7 \text {-Tetramethyltricyclo[5.3.1.0(4.11)] } \\
\text { undec-2-ene }\end{array}$ & 32 & 0.171 & 0.312 & 0.386 & Sesquiterpene & $\mathrm{C}_{15}^{15} \mathrm{H}_{24}^{24}$ \\
\hline 21 & $\begin{array}{l}\text { Decahydro-1,1,3a-trimethyl-7-methylene-[1aS- } \\
(1 \mathrm{a} \alpha, 3 a \alpha, 7 a \alpha, 7 b)]-1 \mathrm{H} \text {-cyclopropa[a]naphthalene }\end{array}$ & 32.153 & 0.37 & 0.034 & - & Sesquiterpene & $\mathrm{C}_{15} \mathrm{H}_{24}$ \\
\hline 22 & 2,2-Bis-(3,5-dimethoxybenzyl)-5,7-dimethoxyindan-1-one & 32.353 & 0.277 & 0.143 & 0.125 & Ketone & $\mathrm{C}_{29} \mathrm{H}_{32} \mathrm{O}_{7}$ \\
\hline 23 & $\begin{array}{l}\text { 4-Ethenyl-4-methyl-3-(1-methylethenyl)-1-(methylethyl)- } \\
\text { (3R-trans)-cyclohexene }\end{array}$ & 32.636 & 1.306 & - & 0.27 & Sesquiterpene & $\mathrm{C}_{15} \mathrm{H}_{24}$ \\
\hline 24 & $\alpha$-Guaiene & 32.847 & 0.092 & 0.959 & 0.65 & Sesquiterpene & $\mathrm{C}_{15} \mathrm{H}_{24}$ \\
\hline 25 & {$[(2,4,6$-Triethylbenzoyl)thio $]$ acetic acid } & 33.096 & - & 0.033 & 0.286 & Acid & $\mathrm{C}_{15}^{13} \mathrm{H}_{20}^{24} \mathrm{O}_{3} \mathrm{~S}$ \\
\hline 26 & (-)-Isoaromadendrene-(V) & 34.045 & 0.189 & 0.118 & 0.108 & Sesquiterpene & $\mathrm{C}_{15}^{15} \mathrm{H}_{24}^{20}$ \\
\hline 27 & $\begin{array}{l}(3 Z, 8 Z)-4,8,11,11 \text {-Tetramethylbicyclo[7.2.0]undeca-3,8- } \\
\text { diene }\end{array}$ & 34.262 & 2.193 & 1.586 & 1.159 & Sesquiterpene & $\mathrm{C}_{15}^{13} \mathrm{H}_{24}^{24}$ \\
\hline 28 & $\begin{array}{l}\text { 1,2,3,3a,4,5,6,7-0ctahydro-1,4-dimethyl-7-(1- } \\
\text { methylethenyl)-[1R-(1 } \alpha, 3 \mathrm{a} \beta, 4 \alpha, 7 \beta)] \text {-azulene }\end{array}$ & 34.513 & 2.862 & - & - & Sesquiterpene & $\mathrm{C}_{15} \mathrm{H}_{24}$ \\
\hline 29 & $\alpha$-Eudesmol & 34.673 & 0.846 & 2.004 & 1.696 & $\begin{array}{l}\text { Oxygenated } \\
\text { sesquiterpene }\end{array}$ & $\mathrm{C}_{15} \mathrm{H}_{24} \mathrm{O}$ \\
\hline 30 & Isocomene & 34.796 & - & 0.215 & 0.062 & Sesquiterpene & $\mathrm{C}_{15} \mathrm{H}_{24}$ \\
\hline 31 & $\begin{array}{l}\text { 1-Ethenyl-1-methyl-2,4-bis(1-methylethenyl)-[1S- } \\
(1 \alpha, 2 \beta, 4 \beta)] \text {-cyclohexane }\end{array}$ & 34.942 & 0.076 & 0.029 & - & Sesquiterpene & $\mathrm{C}_{15}^{15} \mathrm{H}_{24}^{24}$ \\
\hline 32 & $\delta$-Selinene & 35.332 & 2.859 & - & 0.06 & Sesquiterpene & $\mathrm{C}_{15} \mathrm{H}_{24}$ \\
\hline 33 & 1,5,9-Trimethyl-1,5,9-cyclododecatriene & 35.488 & - & 1.733 & 1.238 & Sesquiterpene & $\mathrm{C}_{15}^{15} \mathrm{H}_{24}^{24}$ \\
\hline 34 & 3-Methyl-2-(2,4-pentadienyl)-(Z)-2-cyclopenten-1-one & 35.888 & 3.328 & 3.039 & 1.372 & Ketone & $\mathrm{C}_{11}^{15} \mathrm{H}_{14}^{24} \mathrm{O}$ \\
\hline 35 & Caryophyllene & 36.104 & 0.072 & - & - & Sesquiterpene & $\mathrm{C}_{15}^{11} \mathrm{H}_{24}^{14}$ \\
\hline 36 & $\begin{array}{l}\text { 1-Ethenyl-1-methyl-2-(1-methylethenyl)-4-(1- } \\
\text { methylethyldene)-cyclohexane }\end{array}$ & 36.191 & 3.558 & 0.081 & 0.039 & Sesquiterpene & $\mathrm{C}_{15}^{15} \mathrm{H}_{24}^{24}$ \\
\hline 37 & $\gamma$-Elemene & 36.509 & 0.096 & 4.336 & 1.883 & Sesquiterpene & $\mathrm{C}_{15} \mathrm{H}_{24}$ \\
\hline 38 & $\alpha$-Acorenol & 37.072 & 1.103 & 0.853 & - & $\begin{array}{l}\text { Oxygenated } \\
\text { sesquiterpene }\end{array}$ & $\mathrm{C}_{15}^{15} \mathrm{H}_{26}^{24} \mathrm{O}$ \\
\hline 39 & $\begin{array}{l}\text { 2,6,6,9-Tetramethyl-(1R,2S, } 7 \mathrm{R}, 8 \mathrm{R}) \text {-tricyclo }[5,4,0,0(2,8)] \\
\text { undec-9-ene }\end{array}$ & 37.36 & - & 1.012 & 0.553 & Sesquiterpene & $\mathrm{C}_{15} \mathrm{H}_{24}$ \\
\hline 40 & $\begin{array}{l}{[1 \mathrm{aR} \text {-( }(\mathrm{a} a \alpha, 4 \mathrm{a} \alpha, 7 \beta, 7 \mathrm{a} \beta, 7 \mathrm{~b} \alpha)] \text {-decahydro-1,1,7-trimethyl- }} \\
\text { 4-methylene-, } 1 \mathrm{H} \text {-cycloprop[e]azulen-7-ol }\end{array}$ & 37.759 & 0.438 & 0.033 & 0.037 & $\begin{array}{l}\text { Oxygenated } \\
\text { sesquiterpene }\end{array}$ & $\mathrm{C}_{15} \mathrm{H}_{24} \mathrm{O}$ \\
\hline 41 & $\begin{array}{l}\text { 2-Isopropenyl-4a,8-dimethyl-1,2,3,4,4a,5,6,7- } \\
\text { octahydronaphthalene }\end{array}$ & 38.313 & 0.186 & 0.901 & 3.871 & Sesquiterpene & $\mathrm{C}_{15} \mathrm{H}_{24}$ \\
\hline 42 & $\begin{array}{l}\text { 1-Methyl-5-methylene-8-(1-methylethyl)-[S-(E.E)]-1.6- } \\
\text { cyclodecadiene }\end{array}$ & 38.437 & 1.259 & 0.125 & 0.531 & Sesquiterpene & $\mathrm{C}_{15} \mathrm{H}_{24}$ \\
\hline 43 & 11-Isopropylidenetricyclo[4-3.1.1(2,5)]undec-3-en-10-one & e 38.656 & 0.659 & 2.049 & 0.73 & $\begin{array}{l}\text { Oxygenated } \\
\text { sesquiterpene }\end{array}$ & $\mathrm{C}_{14} \mathrm{H}_{18} \mathrm{O}$ \\
\hline 44 & $\begin{array}{l}\text { 1,5,5,8a-Tetramethyl-decahydro-[1S- } \\
(1 \alpha, 2 \alpha, 3 a \beta, 4 \alpha, 8 a \beta, 9 R)]-1,2,4 \text {-methenoazulne }\end{array}$ & 38.935 & - & 0.318 & 0.356 & Sesquiterpene & $\mathrm{C}_{15} \mathrm{H}_{24}$ \\
\hline 45 & $\beta$-Curcumene & 39.314 & - & 0.078 & - & Sesquiterpene & $\mathrm{C}_{15} \mathrm{H}_{24}$ \\
\hline
\end{tabular}


Table 1: (Continued)

\begin{tabular}{|c|c|c|c|c|c|c|c|}
\hline \multirow[t]{2}{*}{ No } & \multirow[t]{2}{*}{ Compounds } & \multirow{2}{*}{$\begin{array}{c}\text { Retention } \\
\text { time } \\
\text { (min) }\end{array}$} & \multicolumn{3}{|c|}{ Peak area (\%) } & \multicolumn{2}{|c|}{ Class of chemical Chemical formula } \\
\hline & & & $\begin{array}{c}\text { Atractylodes } \\
\text { lancea } \\
\text { Thunb }\end{array}$ & $\begin{array}{l}\text { Atractylodes } \\
\text { japonica } \\
\text { Koidz }\end{array}$ & $\begin{array}{l}\text { Atractylodes } \\
\text { chinensis } \\
\text { Koidz }\end{array}$ & & \\
\hline 46 & $\beta$-Bisabolene & 39.438 & 0.141 & 0.055 & 0.112 & Sesquiterpene & $\mathrm{C}_{15} \mathrm{H}_{24}$ \\
\hline 47 & $\beta$-Copaene & 39.56 & - & - & 0.309 & Sesquiterpene & $\mathrm{C}_{15}^{15} \mathrm{H}_{24}^{24}$ \\
\hline 48 & Isocaryophillene & 39.831 & - & 0.054 & 0.032 & Sesquiterpene & $\mathrm{C}_{15} \mathrm{H}_{24}$ \\
\hline 49 & 3-(1,5-Dimethyl-4-hexenyl)-6-methylene cyclohexene & 39.923 & - & 1.238 & 0.566 & Sesquiterpene & $\mathrm{C}_{15} \mathrm{H}_{2}$ \\
\hline 50 & Cubedol & 40.103 & 0.993 & - & - & $\begin{array}{l}\text { Oxygenated } \\
\text { sesquiterpene }\end{array}$ & $\mathrm{C}_{15} \mathrm{H}_{26} \mathrm{O}$ \\
\hline 51 & Guaia-3,9-diene & 40.434 & 2.785 & - & 2.937 & Sesquiterpene & $\mathrm{C}_{15} \mathrm{H}_{24}$ \\
\hline 52 & Selina-3,7(11)-diene & 40.524 & - & 6.62 & 0.803 & Sesquiterpene & $\mathrm{C}_{15} \mathrm{H}$ \\
\hline 53 & $\begin{array}{l}\text { 2-(3-Isopropyl-4-methyl-pent-3-en-1-ynyl)-2-methyl- } \\
\text { cyclobutanone }\end{array}$ & 40.766 & - & 0.123 & 0.053 & $\begin{array}{l}\text { Oxygenated } \\
\text { sesquiterpene }\end{array}$ & $\mathrm{C}_{14} \mathrm{H}_{20} \mathrm{O}$ \\
\hline 54 & $\gamma$-Himachalene & 40.832 & 0.343 & 0.605 & 0.157 & Sesquiterpene & $\mathrm{C}_{15} \mathrm{H}_{24}$ \\
\hline 55 & Dehydroaromadendrene & 41.318 & 0.373 & 1.478 & 1.425 & Sesquiterpene & $\mathrm{C}_{15} \mathrm{H}_{24}^{24}$ \\
\hline 56 & Calarene epoxide & 41.593 & 0.582 & 1.036 & 0.396 & $\begin{array}{l}\text { Oxygenated } \\
\text { sesquiterpene }\end{array}$ & $\mathrm{C}_{15} \mathrm{H}_{24} \mathrm{O}$ \\
\hline 57 & 1-Hydroxy-1,7,7-dimethyl-4-isopropyl-2,7-cyclodecadiene & 42.3 & 0.576 & 0.224 & 0.265 & $\begin{array}{l}\text { Oxygenated } \\
\text { sesquiterpene }\end{array}$ & $\mathrm{C}_{15} \mathrm{H}_{26} \mathrm{O}$ \\
\hline 58 & $\begin{array}{l}\text { Decahydro-1,5,5,8a-tetramethyl-[1s- } \\
(1 \alpha, 3 a \beta, 4 a \alpha, 7 \beta, 8 a \beta)]-1,4 \text {-methanoazulen-7-ol }\end{array}$ & 42.521 & - & 0.084 & - & $\begin{array}{l}\text { Oxygenated } \\
\text { sesquiterpene }\end{array}$ & $\mathrm{C}_{15} \mathrm{H}_{26} \mathrm{O}$ \\
\hline 59 & Dihydrocucurbitacin B & 42.706 & 0.331 & 0.176 & 0.312 & $\begin{array}{l}\text { Oxygenated } \\
\text { Triterpenoids }\end{array}$ & $\mathrm{C}_{32} \mathrm{H}_{48} \mathrm{O}_{8}$ \\
\hline 60 & Epiglobulol & 43.201 & 0.296 & 0.06 & 0.196 & $\begin{array}{l}\text { Oxygenated } \\
\text { sesquiterpene }\end{array}$ & $\mathrm{C}_{15} \mathrm{H}_{26} \mathrm{O}$ \\
\hline 61 & $\begin{array}{l}\text { 2,2,6-Trimethyl-1-(3-methyl-1,3-butadienyl)-5-methylene- } \\
\text { 7-oxabicyclo[4.1.0]heptane }\end{array}$ & 43.464 & - & 0.191 & 0.104 & $\begin{array}{l}\text { Oxygenated } \\
\text { sesquiterpene }\end{array}$ & $\mathrm{C}_{15} \mathrm{H}_{22} \mathrm{O}$ \\
\hline 62 & Cubenol & 43.558 & 0.092 & - & - & $\begin{array}{l}\text { Oxygenated } \\
\text { sesquiterpene }\end{array}$ & $\mathrm{C}_{15} \mathrm{H}_{26} \mathrm{O}$ \\
\hline 63 & 4-epi-Cubedol & 43.863 & 0.118 & - & 0.081 & $\begin{array}{l}\text { Oxygenated } \\
\text { sesquiterpene }\end{array}$ & $\mathrm{C}_{15} \mathrm{H}_{26} \mathrm{O}$ \\
\hline 64 & Guaiol & 44.138 & 5.006 & - & 0.075 & $\begin{array}{l}\text { Oxygenated } \\
\text { sesquiterpene }\end{array}$ & $\mathrm{C}_{15} \mathrm{H}_{26} \mathrm{O}$ \\
\hline 65 & $\gamma$-Eudesmol & 44.354 & 0.325 & 0.33 & - & $\begin{array}{l}\text { Oxygenated } \\
\text { sesquiterpene }\end{array}$ & $\mathrm{C}_{15} \mathrm{H}_{26} \mathrm{O}$ \\
\hline 66 & $\begin{array}{l}\text { Decahydro- } \alpha, \alpha, 4 a \text {-trimethyl-8-methylene-[2R- } \\
(2 \alpha, 4 \mathrm{a} \alpha, 8 \mathrm{a} \beta)]-2 \text {-naphthalenemethanol }\end{array}$ & 44.608 & 3.62 & 0.153 & 2.515 & $\begin{array}{l}\text { Oxygenated } \\
\text { sesquiterpene }\end{array}$ & $\mathrm{C}_{15} \mathrm{H}_{26} \mathrm{O}$ \\
\hline 67 & Curzerene & 44.991 & 14.179 & 3.558 & 16.729 & $\begin{array}{l}\text { Oxygenated } \\
\text { sesquiterpene }\end{array}$ & $\mathrm{C}_{15} \mathrm{H}_{20} \mathrm{O}$ \\
\hline 68 & $\begin{array}{l}\text { 1,2,3,4,4a,5,6,8a-0ctahydro- } \alpha, \alpha, 4 a, 8 \text {-tetramethyl-[2R- } \\
(2 \alpha, 4 A \alpha, 8 A \beta)]-2 \text {-naphthalenemethanol }\end{array}$ & 45.199 & 1.225 & 0.288 & - & $\begin{array}{l}\text { Oxygenated } \\
\text { sesquiterpene }\end{array}$ & $\mathrm{C}_{15} \mathrm{H}_{26} \mathrm{O}$ \\
\hline 69 & $\begin{array}{l}\text { 3-Hydroxy-7-isopropenyl-1,4a-dimethyl-2,3,4,4a,5,6,7,8- } \\
\text { octahydronaphthalen-2-yl acetate }\end{array}$ & 45.454 & - & 0.568 & - & Ester & $\mathrm{C}_{17} \mathrm{H}_{26} \mathrm{O}_{3}$ \\
\hline 70 & Fenretinide & 45.713 & - & 0.214 & 0.762 & Other & $\mathrm{C}_{26} \mathrm{H}_{33} \mathrm{NO}_{2}$ \\
\hline 71 & Ambrosin & 45.795 & 0.412 & 0.079 & 1.271 & $\begin{array}{l}\text { Oxygenated } \\
\text { sesquiterpene }\end{array}$ & $\mathrm{C}_{15} \mathrm{H}_{18} \mathrm{O}_{3}$ \\
\hline 72 & Diepicedrene-1-oxide & 46.31 & - & 0.091 & 0.137 & $\begin{array}{l}\text { Oxygenated } \\
\text { sesquiterpene }\end{array}$ & $\mathrm{C}_{15} \mathrm{H}_{24} \mathrm{O}$ \\
\hline 73 & $\begin{array}{l}\text { 6-Isopropenyl-4,8a-dimethyl-1,2,3,5,6,7,8,8a- } \\
\text { octahydronaphthalene-2,3-diol }\end{array}$ & 46.482 & 1.817 & - & 1.216 & $\begin{array}{l}\text { Oxygenated } \\
\text { sesquiterpene }\end{array}$ & $\mathrm{C}_{15} \mathrm{H}_{24} \mathrm{O}_{2}$ \\
\hline 74 & [1,1'-Biphenyl]-4-carbonyl chloride & 46.916 & 0.079 & 0.028 & 0.148 & Other & $\mathrm{C}_{13} \mathrm{H}_{9} \mathrm{ClO}$ \\
\hline 75 & Ledene oxide-(II) & 47.443 & - & - & 0.185 & $\begin{array}{l}\text { Oxygenated } \\
\text { sesquiterpene }\end{array}$ & $\mathrm{C}_{15} \mathrm{H}_{24} \mathrm{O}$ \\
\hline 76 & Murolan-3,9(11)-diene-10-peroxy & 47.604 & 0.658 & 16.883 & 0.992 & $\begin{array}{l}\text { Oxygenated } \\
\text { sesquiterpene }\end{array}$ & $\mathrm{C}_{15} \mathrm{H}_{24} \mathrm{O}_{2}$ \\
\hline 77 & Longiverbenone & 47.747 & - & - & 0.031 & $\begin{array}{l}\text { Oxygenated } \\
\text { sesquiterpene }\end{array}$ & $\mathrm{C}_{15} \mathrm{H}_{22} \mathrm{O}$ \\
\hline 78 & Neocurdione & 48.044 & 0.239 & 0.778 & - & $\begin{array}{l}\text { Oxygenated } \\
\text { sesquiterpene }\end{array}$ & $\mathrm{C}_{15} \mathrm{H}_{24} \mathrm{O}_{2}$ \\
\hline 79 & {$\left[1,1^{\prime}\right.$-Biphenyl]-4-carboxaldehyde } & 48.798 & 0.51 & - & 2.647 & Aldehyde & $\mathrm{C}_{13} \mathrm{H}_{10} \mathrm{O}$ \\
\hline 80 & $\begin{array}{l}(3 \beta, 5 Z, 7 E)-9,10-S e c o c h o l e s t a-5,7,10(19) \text {-triene-3,24,25- } \\
\text { triol }\end{array}$ & -49.388 & 0.227 & - & 0.937 & Other & $\mathrm{C}_{27} \mathrm{H}_{44} \mathrm{O}_{3}$ \\
\hline 81 & $\begin{array}{l}\text { 2-Methyl-4-(1,3,3-trimethyl-7-oxabicyclo[4.1.0]hept-2- } \\
\text { yl)-3-buten-2-ol }\end{array}$ & 49.676 & 0.275 & 0.066 & 0.056 & $\begin{array}{l}\text { Oxygenated } \\
\text { sesquiterpene }\end{array}$ & $\mathrm{C}_{14} \mathrm{H}_{24} \mathrm{O}_{2}$ \\
\hline
\end{tabular}




\begin{tabular}{|c|c|c|c|c|c|c|c|}
\hline \multirow[t]{2}{*}{ No } & \multirow[t]{2}{*}{ Compounds } & \multirow{2}{*}{$\begin{array}{c}\text { Retention } \\
\text { time } \\
\text { (min) }\end{array}$} & \multicolumn{3}{|c|}{ Peak area (\%) } & \multicolumn{2}{|c|}{ Class of chemical Chemical formula } \\
\hline & & & $\begin{array}{c}\text { Atractylodes } \\
\text { lancea } \\
\text { Thunb }\end{array}$ & $\begin{array}{c}\text { Atractylodes } \\
\text { japonica } \\
\text { Koidz }\end{array}$ & $\begin{array}{c}\text { Atractylodes } \\
\text { chinensis } \\
\text { Koidz }\end{array}$ & & \\
\hline 82 & $\begin{array}{l}\text { Decahydro-6,9a-dimethyl-3-methylene-, } \\
\text { (3aS,6S,6aS,9aR,9bR)-azuleno[4,5-b]furan-2,9-dione }\end{array}$ & 50.251 & 0.385 & - & - & $\begin{array}{l}\text { Oxygenated } \\
\text { sesquiterpene }\end{array}$ & $\mathrm{C}_{15} \mathrm{H}_{20} \mathrm{O}_{4}$ \\
\hline 83 & trans-Longipinocarveol & 50.734 & 0.163 & 0.032 & 0.024 & $\begin{array}{l}\text { Oxygenated } \\
\text { sesquiterpene }\end{array}$ & $\mathrm{C}_{15} \mathrm{H}_{24} \mathrm{O}$ \\
\hline 84 & $\begin{array}{l}\text { 7,8-Di(hydroxymethyl)-5-methyl-2-isopropyl-spiro-6- } \\
\text { (bicyclo[3.2.1]octane)-2'-(oxirane) }\end{array}$ & 51.739 & 0.132 & 0.109 & - & $\begin{array}{l}\text { Oxygenated } \\
\text { sesquiterpene }\end{array}$ & $\mathrm{C}_{15} \mathrm{H}_{26} \mathrm{O}_{3}$ \\
\hline 85 & $\begin{array}{l}\text { (10a-Hydroxy-3a-methoxy-2,10-dimethyl-3,8-dioxo- } \\
4,6 a, 7,9,10,10 b \text {-hexahydrobenzo[e]azulen-5-yl)methyl } \\
\text { acetate }\end{array}$ & 52 & 2.803 & - & 0.355 & Ester & $\mathrm{C}_{20} \mathrm{H}_{26} \mathrm{O}_{6}$ \\
\hline 86 & $\begin{array}{l}\text { 7,8,15,16-Tetramethyl-1,9-dioxacyclohexadeca-4,13- } \\
\text { diene-2-10-dione }\end{array}$ & 52.709 & 0.126 & 0.394 & 0.72 & Ketone & $\mathrm{C}_{18} \mathrm{H}_{28} \mathrm{O}_{4}$ \\
\hline 87 & $\begin{array}{l}\text { 5-Methyl-1-[2,6,6-trimethyl-2,4-cyclohexadien-1-yl]-1,4- } \\
\text { hexadien-3-one }\end{array}$ & 52.955 & 3.01 & 0.024 & 0.017 & Ketone & $\mathrm{C}_{20} \mathrm{H}_{30} \mathrm{O}_{5}$ \\
\hline 88 & $n$-Hexadecanoic acid & 53.577 & 0.289 & 0.036 & 0.039 & Acid & $\mathrm{C}_{16} \mathrm{H}_{32} \mathrm{O}_{2}$ \\
\hline 89 & Methyl Retinoate & 55.155 & 0.197 & 0.086 & - & Ester & $\mathrm{C}_{21} \mathrm{H}_{30} \mathrm{O}_{2}$ \\
\hline 90 & Andrographolide & 55.555 & 0.278 & - & 0.016 & Ketone & $\mathrm{C}_{16} \mathrm{H}_{22} \mathrm{O}$ \\
\hline 91 & 4,4'-Dimethyl-2,2'-dimethylenebicyclohexyl-3,3'-diene & 56.561 & 0.248 & 0.374 & 0.083 & Alkene & $\mathrm{C}_{16}^{16} \mathrm{H}_{22}^{22}$ \\
\hline 92 & $\begin{array}{l}\text { (6a,9-Dihydroxy-6-methyl-3-methylidene-2-oxo- } \\
3 a, 4,5,6,7,8,9,9 b \text {-octahydroazuleno[4,5-b]furan-9a-yl) } \\
\text { methyl acetate }\end{array}$ & 57.98 & 0.067 & 0.149 & 0.037 & Ester & $\mathrm{C}_{17} \mathrm{H}_{24} \mathrm{O}_{6}$ \\
\hline 93 & Propoxyphene & 61.099 & 1.077 & - & 0.019 & Ketone & $\mathrm{C} 22 \mathrm{H} 2906$ \\
\hline 94 & 9,10-dihydro-9,10[1',2']-benzenoanthracene & 64.218 & 0.042 & 4.39 & 0.12 & Alkene & $\mathrm{C}_{20} \mathrm{H}_{14}$ \\
\hline 95 & 3-(4-Methoxyphenyl)-2-ethylhexyl-2-propenoate & 64.58 & 0.249 & 1.622 & 1.079 & Ester & $\mathrm{C}_{18}^{20} \mathrm{H}_{26} \mathrm{O}_{3}$ \\
\hline 96 & 2-(4-Diethylaminophenyliminomethyl)phenol & 66.929 & 0.154 & 1.272 & 3.264 & Acid & $\mathrm{C}_{17} \mathrm{H}_{20} \mathrm{~N}_{2}$ \\
\hline 97 & 9-Cycloheptatrienylidene-9,10-dihydro-10-oxoanthracene & 74.656 & - & 1.87 & 0.178 & Ketone & $\mathrm{C}_{21}^{11} \mathrm{H}_{14} \mathrm{O}^{2}$ \\
\hline 98 & Methyl 9,11-octadecadienoate & 78.213 & - & 5.221 & - & Ester & $\mathrm{C}_{19} \mathrm{H}_{30} \mathrm{O}_{2}$ \\
\hline \multirow[t]{2}{*}{99} & $\begin{array}{l}\mathrm{N} \text {-[[3,6-dichloro-2,7-bis(2-diethylaminoethyloxy)fluoren- } \\
\text { 9-ylidene]amino]-2,2-dimethylpropanamide }\end{array}$ & 78.587 & 0.669 & 9.382 & - & Other & $\mathrm{C}_{30} \mathrm{H}_{42} \mathrm{C}_{12} \mathrm{~N}_{4} \mathrm{O}_{3}$ \\
\hline & & Total & 82.528 & 81.766 & 81.799 & & \\
\hline
\end{tabular}

7,8-di(hydroxymethyl)-5-methyl-2-isopropyl-spiro6-(bicyclo[3.2.1] octane)-2'-(oxirane), methyl retinoate, and $N$-[ [3,6-dichloro-2,7-bis(2-diethylaminoethyloxy) fluoren-9-ylidene]amino]-2,2-dimethylpropanamide were observed both in A. lancea and A. japonica samples. Both in A. japonica and A. chinensis further 14 compounds were detected, namely $\gamma$-terpinene, [(2,4,6-triethylbenzoyl) thio]acetic acid, isocomene, 1,5,9-trimethyl-1,5,9cyclododecatriene, 2,6,6,9-tetramethyl-(1R,2S,7R,8R)tricyclop $[5,4,0,0(2,8)]$ undec-9-ene, $1,5,5,8$ a-tetramethyldecahydro-[1S-(1 $\alpha, 2 \alpha, 3 \mathrm{a} \beta, 4 \alpha, 8 \mathrm{a} \beta, 9 \mathrm{R})]-1,2,4-$ methenoazulne, isocaryophillene, 3-(1,5-dimethyl-4-hexenyl)-6-methylenecyclohexene, selina-3,7(11)-diene, 2-(3-isopropyl-4-methylpent-3-en-1-ynyl)-2-methyl-cyclobutanone, 2,2,6-trimethyl1-(3-methyl-1,3-butadienyl)-5-methylene-7-oxabicyclo[4,1,0] heptane, fenretinide, diepicedrene-1-oxide, and 9-cycloheptatrienylidene-9,10-dihydro-10-oxoanthracene. The remaining compounds, i.e. D-limonene, $\alpha$-ylangene, 4-terpinenyl acetate, 1,5,5,6-tetramethyl-1,3-cyclohexadiene, 1,2-ethanediol monobenzoate, 2,6-pyridinedicarboxaldehyde, 3,6-diethyl-3,6-dimethyl-trans-tricyclo[3.1.0.0(2.4)] hexane, longifolene-(V4), 4-ethenyl-4-methyl-3-(1-methylethenyl)-1(methylethyl)-(3R-trans)-cyclohexene, $\delta$-selinene, guaia-3,9diene, 4-epi-cubedol, guaiol, 6-isopropenyl-4,8a-dimethyl1,2,3,5,6,7,8,8a-octahydronaphthalene-2,3-diol, [1,l'-biphenyl]4-carboxaldehyde, (3ß,5Z,7E)-9,10-secocholesta-5,7,10(19)triene-3,24,25-triol, (10a-hydroxy-3a-methoxy-2,10-dimethyl- 3,8-dioxo-4,6a, 7,9,10,10b-hexahydrobenzo[e] azulen-5-yl) methyl acetate, andrographolide, and propoxyphene, were detected both in A. lancea and A. chinensis. Considering all 99 volatile compounds, the total amounts contained in each of the three Atractylode species were comparable, with values of $82.528 \%, 81.766 \%$, and $81.799 \%$ calculated for A. lancea, A. japonica, and the most abundant volatile and A. chinesis, respectively. Among these compounds detected in A. lancea were curzerene $(14.1 \%)$ and 3-octen-5-yne $(9.01 \%)$, while murolan3,9(11)-diene-10-peroxy (16.8\%) was the most abundant volatile in A. japonica. The most abundant compounds in A. chinensis were curzerene (16.7\%), $\gamma$-terpinene (10.3\%), $\mathrm{N}$-[ [3,6-dichloro2,7-bis(2-diethylaminoethyloxy)fluoren-9-ylidene]amino]-2,2dimethylpropanamide (9.4\%), and 4-terpinenyl acetate (8.2\%).

\section{Numbers of Volatile Flavor Compounds and their Quantities (\%) in Different species of Atractylodes}

There are 13 volatile flavor compounds i.e. acid, alcohol, aldehyde, alkane, alkene, alkyne, ester, ketone, monoterpene, oxygenated monoterpene, sesquiterpene, oxygenated sesquiterpene, and oxygenated triterpenoid detected from different species of Atractylodes (Table 2). The amount of alcohol was too low within the species where no alcohol was found in A. chinensis. The highest amount of acid, aldehyde, alkane, monoterpene, and oxygenated monoterpene types volatile flavor compounds were 
Table 2: Numbers of volatile flavor compounds in Atractylodes spp.

\begin{tabular}{|c|c|c|c|c|c|c|}
\hline \multirow[t]{2}{*}{$\begin{array}{l}\text { Class of } \\
\text { chemical } \\
\text { components }\end{array}$} & \multicolumn{2}{|c|}{$\begin{array}{c}\text { Atractylodes } \\
\text { lancea } \\
\text { Thunb }\end{array}$} & \multicolumn{2}{|c|}{$\begin{array}{c}\text { Atractylodes } \\
\text { japonica } \\
\text { Koidz }\end{array}$} & \multicolumn{2}{|c|}{$\begin{array}{l}\text { Atractylodes } \\
\text { chinensis } \\
\text { Koidz }\end{array}$} \\
\hline & Number & $\%$ & Number & $\%$ & Number & $\%$ \\
\hline Acid & 2 & 0.443 & 3 & 1.341 & 3 & 3.589 \\
\hline Alcohol & 1 & 0.171 & 1 & 0.135 & 1 & - \\
\hline Aldehyde & 2 & 0.665 & 0 & - & 0 & 2.949 \\
\hline Alkane & 1 & 0.193 & 0 & - & 1 & 0.647 \\
\hline Alkene & 3 & 0.799 & 2 & 4.764 & 2 & 0.203 \\
\hline Alkyne & 1 & 9.007 & 0 & - & 0 & - \\
\hline Ester & 6 & 3.498 & 6 & 8.599 & 5 & 2.904 \\
\hline Ketone & 6 & 8.096 & 5 & 5.470 & 7 & 2.447 \\
\hline Monoterpene & 4 & 3.194 & 3 & 0.718 & 7 & 12.796 \\
\hline $\begin{array}{l}\text { Oxygenated } \\
\text { monoterpene }\end{array}$ & 1 & 0.463 & 0 & - & 1 & 8.205 \\
\hline Sesquiterpene & 20 & 19.911 & 21 & 21.887 & 24 & 18.400 \\
\hline $\begin{array}{l}\text { Oxygenated } \\
\text { sesquiterpene }\end{array}$ & 23 & 34.139 & 21 & 29.024 & 20 & 26.789 \\
\hline $\begin{array}{l}\text { Oxygenated } \\
\text { triterpenoid }\end{array}$ & 1 & 0.331 & 1 & 0.176 & 1 & 0.312 \\
\hline Other & 4 & 1.618 & 4 & 9.652 & 4 & 2.558 \\
\hline Total & 75 & 82.528 & 67 & 81.766 & 76 & 81.799 \\
\hline
\end{tabular}

found in A. chinensis. The levels of accumulation of alcohol, alkyne, ketone, and oxygenated sesquiterpenes were found to be the highest amount in A. lancea. The species A. japonica contained the highest amount of alkene, ester and sesquiterpene. Among the volatile flavor compounds oxygenated sesquiterpene dominated over other volatile flavors compounds irrespective of species. The species A. lancea, A. japonica, and A. chinensis contained 23, 21, and 20 oxygenated sesquiterpenes, having $41.37 \%, 35.50 \%$, and $32.75 \%$ of total volatile flavor compounds, respectively. After oxygenated sesquiterpene, the second-largest accumulated volatile flavor compounds were sesquiterpene. Here the contained of sesquiterpene was $26.77 \%, 24.13 \%$, and $22.49 \%$ in the A. japonica, A. lancea, and A. chinensis, respectively. Volatile flavor compounds alkyne was detected only in the species of A. lancea having $10.91 \%$ of total volatile flavor compounds. The amount of ester was $10.52 \%, 4.24 \%$, and $3.55 \%$ in the A. japonica, A. lancea, and A. chinensis, respectively.

\section{DISCUSSION}

The essential oils and volatile compounds derived from the A. lancea, A. japonica, and A. chinensis species have been reported to have therapeutic value in Chinese medicine. The present study identified 99 volatile compounds from these plants using GC-MS, by quantifying each volatile compound in the three species. Interestingly, it was found that although all extracts contained 38 common compounds, each extract also contained volatiles unique to that particular species. It was found that the variation in the quantity of these compounds depends on the location of the collected samples as well as the differences of species. A total of 77 volatile compounds were detected in total having 13 monoterpenoids, 19 sesquiterpenoids, and others in Mentha species (Park et al., 2016). In an another study (Zouaoui et al., 2020) reported that a total of 91 volatile organic compounds (VOC): 39
VOC were identified in Thymusalgeriensis (with dominance of $\beta$-myrcene $=13.78 \%$, camphor $=12.29 \%$, linalyl acetate $=9.11 \%) ; 37 \mathrm{VOC}$ in Artemisiacampestris $(\beta$-farnesene $=$ $14.17 \%, \beta$-myrcene $=13.84 \%) ; 50 \mathrm{VOC}$ in Juniperusphoenicea $(\alpha$-pinene $=27.18 \%) ; 42 \mathrm{VOC}$ in Teucrium polium $(\alpha$-guaiene $=11.33 \%$, trans-caryophyllene $=9.49 \%, \gamma$-elemene $=9.25 \%$ ), $45 \mathrm{VOC}$ in Rosmarinus officinalis (camphor $=17.46 \%$, transcaryophyllene $=14.83 \%$ ); and 41 in Artemisia herba-alba $(\alpha$-thujone $=24.59 \%, \beta$-thujone $=13.73 \%)$. In Artemisia herba-alba growing in the region of biskra, $\alpha$-thujone $(24.59 \%)$ and $\beta$-thujone (13.73\%) were the major compounds, followed by verbenene $(8.30 \%)$, sabinol $(7.51 \%)$, carvone $(5.05 \%)$, and p-cineole $(4.81 \%)$. These results are partially similar to those reported by (Belhattab et al., 2014) that used plant samples from different regions (Benifouda, Bougaa, Boussaada, and Boutaleb) of Algeria; and from the region of Buseirah (Jordan) (Abu-Darwish et al., 2015). Here in this study, a total of 99 different volatile compounds have been detected which indicated variation of volatile compounds might vary with the variation of region. The nature of volatile compounds varied from species to species. $\alpha$-pinene $(27.18 \%)$ was the major compound in Juniperus phoenicea growing in drylands of Algeria. It is followed by $\beta$-citronellol (6.13\%), $\delta$-3-carene (4.78\%), $\beta$-farnesene (4.71\%), $\alpha$-terpineol (4.12\%), germacrene D $(3.50 \%), \delta$-cadinene $(3.26 \%)$, and geranyl acetone $(3.01 \%)$. These results are in agreement with those described by (Mazari et al., 2010) in the region of Sidi Safi (Tlemcen, Algeria) and in the region of Angad (Oujda, Morocco) (Ait-Ouazzou et al., 2012).The major VOC in Teucriumpolium were $\alpha$-guaiene (11.33\%), trans-caryophyllene (9.49\%), and $\gamma$-elemene (9.25\%). These are followed by $\beta$-farnesene (7.56\%), farnesol (6.14\%), allo-aromadendrene $(4.34 \%), \delta$-guaiene $(4.21 \%)$, geranyl acetone $(3.65 \%)$ and $\alpha$-gurjunene (3.36\%). Octyl acetate (24.22 to $33.16 \%$ ), 2-undecanone (12.43 to $23.82 \%$ ), and 2-nonanone (14.11 to $41.69 \%$ ) were found to be major components of the volatiles extracted by hydro distillation or head-space method of two populations of Ruta chalepensis L. (Rutaceae) (Fakhfakh et al., 2012), whereas in this study curzerene was found to be the most predominant compound in both A. lancea (14.1\%) and A. chinensis (16.7\%), while murolan-3,9(11)-diene-10peroxy was found predominantly in A. japonica (16.8\%). Our findings are in agreement with many previous studies that applied the same procedures for the extraction and detection of volatile compounds. For instance, when GC-MS was used, the number of VOC was 61 compounds in Teucrium polium (Gholivand et al., 2013) and 42 compounds in Rhaponticum acaule roots (Benyelles et al., 2014). The chemical composition of Thymus algeriensis is marked by the presence of $\beta$-myrcene $(13.78 \%)$, camphor $(12.29 \%)$, and linalyl acetate $(9.11 \%)$ as the major constituents, followed by p-cineole $(6.31 \%), \beta$-farnesene $(5.23 \%)$, terpineol $(5.07 \%)$, bornyl acetate $(4.79 \%), \alpha$-pinene $(4.65 \%)$ and camphene $(4.61 \%)$. These results are partially in line with those reported by (Zouari et al., 2011) and (Ali et al., 2012). According to (Ali et al., 2015), there is a large quantitative and qualitative variation in VOC between leaves, stems, and roots of the same plant species. In Artemisia campestris growing in Algerian drylands, the major VOC are $\beta$-farnesene $(14.17 \%)$ and $\beta$-myrcene $(13.84 \%)$ followed by $\alpha$-cedrene (7.88\%), germacrene D (7.29\%), $\alpha$-pinene (4.63\%), 
and $\beta$-pinene (4.21\%). These results are partially in line with those reported by (Ghorab et al., 2013) and (Al Jahid et al., 2016). The slight quantitative difference in contents of major VOC may be due to genetic variation and geographical origin of plant material; knowing that (Al Jahid et al., 2016) collected samples from Saharan zones of Morocco, whereas (Ghorab et al., 2013) harvested plants from semi-arid areas of Algeria. Besides, differences in VOC contents between studies can be related to differences in extraction method, analysis conditions or even the vegetal organ analyzed 'leaves in (Al Jahid et al., 2016)' or the freshness of plant materials, as (Ghorab et al., 2013) used fresh plants in VOC screening while most studies use dried plant materials.

The present study suggests that the identified volatile compounds may possess important biological properties, and could be suitable for application in both oriental medicines and the pharmaceutical industry. This report, therefore, presents further information regarding the quantification and abundance of these volatile compounds, which are expected to possess a range of important biological properties, and could there be useful for application in oriental medicine in countries such as Korea and China.

\section{CONCLUSION}

Based on these results, it is suggested that the Atractylodes species and their identified volatile compounds may possess important biological properties, and could be suitable for application in both oriental medicines and in the pharmaceutical industry. Appropriate separation of the components within these essential oils may lead to the development of new drug targets or therapeutic treatments.

\section{REFERENCES}

Abu-Darwish, M., Cabral, C., Gonçalves, M., Cavaleiro, C., Cruz, M., Efferth, T., and Salgueiro, L. (2015). Artemisia herba-alba essential oil from Buseirah (South Jordan): Chemical characterization and assessment of safe antifungal and anti-inflammatory doses. Journal of Ethnopharmacology, 174, 153-160. https://doi.org/10.1016/j. jep.2015.08.005

Ait-Ouazzou, A., Lorán, S., Arakrak, A., Laglaoui, A., Rota, C., Herrera, A., Pagán, R., and Conchello, P. (2012). Evaluation of the chemical composition and antimicrobial activity of Mentha pulegium, Juniperus phoenicea, and Cyperus longus essential oils from Morocco. Food Research International, 45(1), 313-319. https://doi.org/10.1016/j. foodres.2011.09.004

Al Jahid, A., Essabaq, S., Elamrani, A., Blaghen, M., and Jamal Eddine, J. (2016). Chemical composition, antimicrobial and antioxidant activities of the essential oil and the hydro-alcoholic extract of Artemisia campestris L. leaves from southeastern Morocco. Journal of Biologically Active Products from Nature, 6(5-6), 393-405. https:// doi.org/10.1080/22311866.2016.1268068

Ali, I. B. E. H., Chaouachi, M., Bahri, R., Chaieb, I., Boussaïd, M., and Harzallah-Skhiri, F. (2015). Chemical composition and antioxidant, antibacterial, allelopathic and insecticidal activities of essential oil of Thymus algeriensis Boiss. et Reut. Industrial Crops and Products, 77. 631-639. https://doi.org/10.1016/j.indcrop.2015.09.046

Ali, I. B. E. H., Guetat, A., and Boussaid, M. (2012). Chemical and genetic variability of Thymus algeriensis Boiss. et Reut. (Lamiaceae), a North African endemic species. Industrial Crops and Products, 40, 277-284. https://doi.org/10.1016/j.indcrop.2012.03.021

Belhattab, R., Amor, L., Barroso, J. G., Pedro, L. G., and Figueiredo, A. C.
(2014). Essential oil from Artemisia herba-alba Asso grown wild in Algeria: Variability assessment and comparison with an updated literature survey. Arabian Journal of Chemistry, 7(2), 243-251. https:// doi.org/10.1016/j.arabjc.2012.04.042

Benyelles, B., Allali, H., Dib, M. E. A., Djabou, N., Tabti, B., and Costa, J. (2014). Essential oil from Rhaponticum acaule L. roots: Comparative study using HS-SPME/GC/GC-MS and hydrodistillation techniques. Journal of Saudi Chemical Society, 18(6), 972-976. https://doi. org/10.1016/j.jscs.2011.12.001

Chen, Q., Li, P., Yang, H., Li, X., Zhu, J., and Chen, F. (2009). Identification of volatile compounds of Atractylode lancea rhizoma using supercritical fluid extraction and GC-MS. Journal of Separation Science, 32(18), 3152-3156. https://doi.org/10.1002/jssc.200900210

Chen, Y., Chou, G., and Wang, Z. (2007). Simultaneous determination of polyacetylene components in Cangzhu by reversed-phase high performance liquid chromatography. Se Pu (Chinese Journal of Chromatography), 25(1), 84-87.

Chu, S. S., Jiang, G. H., and Liu, Z. L. (2011). Insecticidal compounds from the essential oil of Chinese medicinal herb Atractylodes chinensis. Pest Management Science, 67, 1253-1257. https://doi.org/10.1002/ ps. 2180

Dudareva, N., Pichersky, E., and Gershenzon, J. (2004). Biochemistry of plant volatiles. Plant Physiology, 135, 1893-1902. https://doi. org/10.1104/pp.104.049981

Fakhfakh, N., Zouari, S., Zouari, M., Loussayef, C., and Zouari, N. (2012). Chemical composition of volatile compounds and antioxidant activities of essential oil, aqueous and ethanol extracts of wild Tunisian Ruta chalepensis L. (Rutacea). Journal of Medicinal Plants Research, 6(4), 593-600. https://doi.org/10.5897/JMPR11.1121

Gholivand, M. B., Piryaei, M., Abolghasemi, M. M., and Maassoumi, S. M. (2013). Rapid analysis of volatile components from Teucrium polium L. by nanoporous silica-polyaniline solid phase microextraction fibre. Phytochemical Analysis, 24, 69-74. https://doi.org/10.1002/pca.2382

Ghorab, H., Laggoune, S., Kabouche, A., Semra, Z., and Kabouche, Z. (2013). Essential oil composition and antibacterial activity of Artemisia campestris L. from Khenchela (Algeria). Der Pharmacia Lettre, 5(2), 189-192.

Guo, F. -Q., Huang, L. -F., Zhou, S. -Y., Zhang, T. -M., and Liang, Y. -Z. (2006). Comparison of the volatile compounds of Atractylodes medicinal plants by headspace solid-phase microextraction-gas chromatography-mass spectrometry. Analytica Chimica Acta, 570(1), 73-78. https://doi.org/10.1016/j.aca.2006.04.006

Mazari, K., Bendimerad, N., and Bekhechi, C. (2010). Chemical composition and antimicrobial activity of essential oils isolated from Algerian Juniperus phoenicea L. and Cupressus sempervirens L. Journal of Medicinal Plants Research, 4(10), 959-964.

Park, Y. J., Baskar, T. B., Yeo, S. K., Arasu, M. V., Al-Dhabi, N. A., Lim, S. S., and Park, S. U. (2016). Composition of volatile compounds and in vitro antimicrobial activity of nine Mentha spp. SpringerPlus, 5(1), 1-10. https://doi.org/10.1186/s40064-016-3283-1

Pichersky, E., Noel, J.P., and Dudareva, N. (2006). Biosynthesis of plant volatiles: nature's diversity and ingenuity. Science, 311(5762), 808811. https://doi.org/10.1126/science.1118510

Ren, C. -G., and Dai, C. -C. (2012). Jasmonic acid is involved in the signaling pathway for fungal endophyte-induced volatile oil accumulation of Atractylodes lancea plantlets. BMC Plant Biology, 12(128), 1-11. https://doi.org/10.1186/1471-2229-12-128

Takeda, O., Miki, E., Terabayashi, S., Okada, M., Lu, Y., He, H. -S., and He, S. -A. (1996). A comparative study on essential oil components of wild and cultivated Atractylodes lancea and $A$. chinensis. Planta Medica, 62(5), 444-449. https://doi.org/10.1055/s-2006-957936

Unsicker, S. B., Kunert, G., and Gershenzon, J. (2009). Protective perfumes: the role of vegetative volatiles in plant defense against herbivores. Current Opinion in Plant Biology, 12(4), 479-485. https://doi. org/10.1016/j.pbi.2009.04.001

Wang, X. M., Yang, B., Ren, C. G., Wang, H. W., Wang, J. Y., and Dai, C. C. (2015). Involvement of abscisic acid and salicylic acid in signal cascade regulating bacterial endophyte-induced volatile oil biosynthesis in plantlets of Atractylodes lancea. Physiologia Plantarum, 153(1), 30-42. https://doi.org/10.1111/ppl.12236

Wang, Y., Dai, C. -C., Cao, J. -L., and Xu, D. -S. (2012). Comparison of the effects of fungal endophyte Gilmaniella sp. and its elicitor on Atractylodes lancea plantlets. World Journal of Microbiology and Biotechnology, 28, 575-584. https://doi.org/10.1007/s11274-011-0850-z 
Willis, J. C. (1966). A dictionary of the flowering plants and ferns. A dictionary of the flowering plants and ferns. Science, 157(3789), 1289. https://doi.org/10.1126/science.157.3789.674-a

Xie, Y. L., Li, Z. M., Huang, M. Q., Su, Z. R., and Su, X. P. (2008). Determination of atrctylodin and atractylone from the rhizomes of Atractylodes lancea by GC. Chinese Traditional and Herbal Drugs, 39, 614-615.

Yosioka, I., Nishino, T., Tani, T., and Kitagawa, I. (1976). On the constituents of the rhizomes of Atractylodes lancea DC var. chinensis KITAMURA ("Jin-changzhu") and Atractylodes ovata DC ("Chinese baizhu"). The gas chromatographic analysis of the crude drug "Zhu".Yakugaku zasshi: Journal of the Pharmaceutical Society of Japan, 96(10), 1229-1235.

Zhang, H., Han, T., Sun, L. -N., Huang, B. -K., Chen, Y. -F., Zheng, H. -C., and Oin, L. -P. (2008). Regulative effects of essential oil from
Atractylodes lancea on delayed gastric emptying in stress-induced rats. Phytomedicine, 15(8), 602-611. https://doi.org/10.1016/j. phymed.2008.02.005

Zouaoui, N., Chenchouni, H., Bouguerra, A., Massouras, T., and Barkat, M. (2020). Characterization of volatile organic compounds from six aromatic and medicinal plant species growing wild in North African drylands. NFS Journal, 18, 19-28. https://doi.org/10.1016/j. nfs. 2019.12.001

Zouari, N., Fakhfakh, N., Zouari, S., Bougatef, A., Karray, A., Neffati, M., and Ayadi, M. (2011). Chemical composition, angiotensin I-converting enzyme inhibitory, antioxidant and antimicrobial activities of essential oil of Tunisian Thymus algeriensis Boiss. et Reut. (Lamiaceae). Food and Bioproducts Processing , 89(4), 257-265. https://doi.org/10.1016/j. fbp.2010.11.006 